\title{
Next-generation sequencing-based Actinobacteria community associated with Callyspongia sp. from Kepulauan Seribu Marine National Park, Jakarta, Indonesia
}

\author{
DWI RETNOWATI ${ }^{1,2}$, DEDY DURYADI SOLIHIN ${ }^{2}$, MUNIF GHULAMAHDI ${ }^{3}$, YULIN LESTARI ${ }^{2,4,4}$ \\ ${ }^{1}$ Sekolah Tinggi Intelijen Negara. Jl. Sumur Batu, Babakan Madang, Bogor 16810, West Java, Indonesia \\ ${ }^{2}$ Department of Biology, Faculty of Mathematics and Natural Sciences, Institut Pertanian Bogor. Jl. Agatis, Campus IPB Dramaga, Bogor 16680, West \\ Java, Indonesia.Tel/fax.: +62-251-8622833. vemail: yulinlestari@gmail.com, retnowatidwi1112@gmail.com \\ ${ }^{3}$ Department Agronomy and Horticulture, Faculty of Agriculture, Institut Pertanian Bogor. Jl. Agatis, Campus IPB Dramaga, Bogor 16680, West Java, \\ Indonesia \\ ${ }^{4}$ Tropical Biopharmaca Research Center, Institut Pertanian Bogor. Jl. Taman Kencana No. 3, Campus IPB Taman Kencana, Bogor 16128, West Java, \\ Indonesia.
}

Manuscript received: 11 June 2021. Revision accepted: 14 August 2021.

\begin{abstract}
Retnowati D, Solihin DD, Ghulamahdi M, Lestari. 2021. Next-generation sequencing-based Actinobacteria community associated with Callyspongia sp. from Kepulauan Seribu Marine National Park, Jakarta, Indonesia. Biodiversitas 22: $3702-3708$. Sponges are sessile benthic filter-feeding animals, which harbor numerous microbes. The enormous community and abundance of sponge-associated actinobacteria envisage sponges as hot spots of microbes. The metagenomic approach with Next Generation Sequencing (NGS) technology was used to investigate the actinobacteria community of Callyspongia sp. collected from Kepulauan Seribu Marine National Park, Indonesia. Fourteen actinobacteria families were found associated with the sponge. Among them, Microbacteriaceae was the most dominant family. The community of actinobacteria was higher than that of the culturable method from our previous study. The research showed that a metagenomic approach using NGS technology reveals better information regarding the actinobacteria community associated with Callyspongia sp.
\end{abstract}

Keywords: Callyspongia, metagenomic approach, sponge-associated actinobacteria

\section{INTRODUCTION}

Sponges are the most ancient Metazoa and generally have strong symbiotic relations with the complex communities of microbes. Actinobacteria were found as one of the bacterial groups associated with 3569 sponge samples obtained from almost all over the world with a relative abundance of 5\% (Silva et al. 2017). Recent evidence suggests that various genera of culturable actinobacteria were obtained to be associated with 50 types of sponges (Abdelmohsen et al. 2014). Intriguingly, members of the phylum actinobacteria have been identified as abundant members of sponge-associated microbial communities.

Research on sponge-associated actinobacteria communities has been widely used culture-dependent and several of them using metagenomic approach (Bibi et al 2019). A metagenomic approach can better reveal all community members of the association because culturable microbes are thought to constitute less than $1 \%$ while the other $99 \%$ are unculturable microbes. On the basis of the metagenomic approach, several methods such as the Denaturing Gradient Gel Electrophoresis (DGGE) and the Terminal Restriction Fragment Length Polymorphism (TRFLP) have been widely applied to determine the community of sponge-associated actinobacteria (Simamora et al. 2016). However, reports on the sponge-associated actinobacteria community by using Next-Generation Sequencing (NGS) technology are still limited. The NGS technology allows gene sequencing from complex environmental samples such as bacterial communities associated with marine sponges, including the analysis of actinobacteria communities in a comprehensive manner (Bokulich et al. 2012). This paper attempts to show the existence of Callyspongia sp. sponge-associated actinobacterial communities using a NGS approach.

Callyspongia sp. is one of the common sponges found in the Kepulauan Seribu Marine National Park, Indonesia. Several reports have shown that Callyspongia sp. has the capability to produce various secondary metabolites, and the active compounds showed cytotoxic activity and acyltransferase inhibitor (Gelani and Uy 2016; Kapojos et al. 2018). Bioactive compounds produced by sponges are thought to be the result of their interactions with associated bacteria. However, the community of actinobacteria associated with this marine sponge from Kepulauan Seribu Marine National Park has not yet been studied further. This study aimed to reveal the actinobacteria community associated with Callyspongia sp. based on a metagenomic approach using a NGS technology. 


\section{MATERIALS AND METHODS}

\section{Sponge collection}

Specimens of the marine sponge Callyspongia sp. were collected at a depth of $16 \mathrm{~m}$ below sea surface of Panggang island Kepulauan Seribu Marine National Park $\left(5^{\circ} 38^{\prime} 00^{\prime \prime}-5^{\circ} 45^{\prime} 00^{\prime \prime} \mathrm{S}\right.$, and $\left.106^{\circ} 33^{\prime} 00^{\prime \prime}-106^{\circ} 47^{\prime} \mathrm{E}\right)$, Jakarta, Indonesia. The samples were rinsed with sterile seawater and placed in sterile plastic trays. All sponges were stored in a freezer box before being examined. All sponges were identified by the Fisheries Diving Club of the Faculty of Fisheries and Marine Science (IPB University).

\section{DNA extraction}

One gram of sponge sample was crushed into powder in liquid nitrogen. The DNA extraction methods utilized Zymobiomics DNA Isolation Mini Kit, following the manufacturer's protocol. The DNA was stored at $-20^{\circ} \mathrm{C}$ until further investigation.

\section{Next-generation sequencing analysis}

The bacterial 16S rRNA gene was used as DNA template for amplification of the V4 and V5 domains, with primers: 515F-907R modified to contain MiSeq Illumina platform (Illumina, Inc., San Diego, CA, USA) according to customer service by Axil Scientific, Singapore. PCR reactions were conducted using the Q5 Hot Start High Fidelity PCR 2X Master Mix (New England Biolabs, Ipswich, Massachusetts, USA) according to the two-step cycling standard protocol recommended by the manufacturer. The corresponding reaction began with the initial denaturation at $98^{\circ} \mathrm{C}$ for 30 seconds followed by 30 cycles of $98^{\circ} \mathrm{C}$ for 10 seconds, 55-65oC of annealing temperature for 30 seconds, $72^{\circ} \mathrm{C}$ for 30 seconds, a final extension at $72^{\circ} \mathrm{C}$ for 2 minutes and, lastly, hold at $4^{\circ} \mathrm{C}$. Furthermore, the second stage PCR reaction that involved the addition of dual indices (Nextera XT i7 index and Nextera XT i5 index) was prepared with total volume of 50 $\mu \mathrm{L}$. This particular reaction was started with an initial denaturation at $98^{\circ} \mathrm{C}$ for 3 minutes, 8 cycles of $98^{\circ} \mathrm{C}$ for 30 seconds, $55^{\circ} \mathrm{C}$ for 30 seconds, $72^{\circ} \mathrm{C}$ for 30 seconds, and a final extension at $72^{\circ} \mathrm{C}$ for 5 minutes was done prior to putting on hold at $4^{\circ} \mathrm{C}$.

\section{Analysis of the sequence data}

Raw fastq files were de-multiplexed, quality filtered, and analyzed with QIIME V1.7.0. The tags obtained from the previous step were compared to the reference database (Gold database, http://drive5.com/uchime/uchime_download.html) using UCHIME algorithm (http://www.drive5.com/ usearch/manual/uchime_algo.html) to detect chimera sequences. Operational Taxonomic Unit (OTU) clustering and analysis were conducted by UPARSE v.7.0.1001 (http://drive5.com/uparse/). Moreover, the sequences were clustered into the OTUs with $\geq 97 \%$ similarity. Sequence frequently existed within each OTUs was chosen and screened to obtain representative sequence. This research focused on actinobacteria at the level of class, order, and family.

\section{RESULTS AND DISCUSSION}

\section{DNA purity of 16S rRNA gene}

DNA purity of Callyspongia sp. produced using the mini DNA Zymobiomics extraction method was 1.85 (UV 260/280). Interestingly, the amplification results discovered several DNA bands that appeared, indicating the isolation process was completely carried out and the sample contained more than one organism (Figure 1). There were more than one band found in the $300 \mathrm{bp}$ molecular weights.

\section{Diversity and taxonomic rank of bacteria associated with Callyspongia sp. sponges}

A total of 107,223 raw sequences was found from Callyspongia sp. after trimming, denoising, and removing chimera sequences with 67,885 sequences were obtained and subjected to downstream analysis. It was discovered 97\% similarities and were clustered into 500 OTUs. They consisted of were 7 different bacterial phyla, i.e. Proteobacteria (82\%), Acidobacteria (12\%), Planctomycete (2\%), Actinobacteria (2\%), Bacteriodetes (1.08\%), Firmicutes (0.61\%), and Cyanobacteria (0.09\%) (Figure 2).

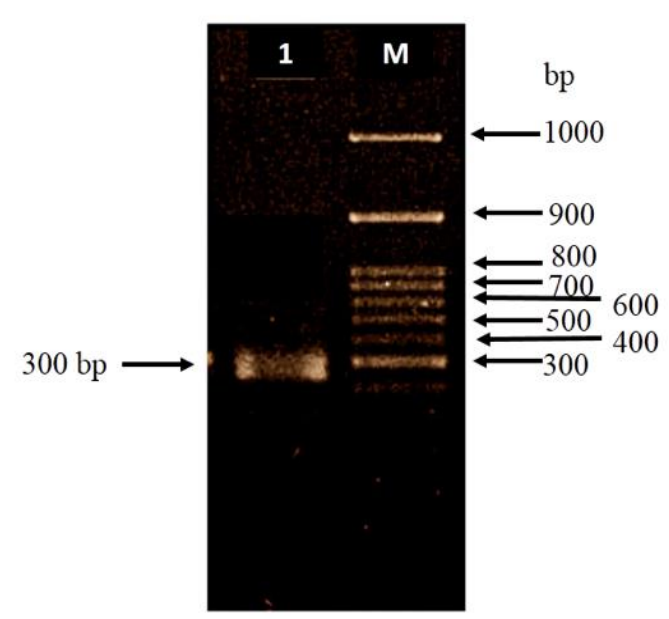

Figure 1. The amplified of $16 \mathrm{~S}$ rRNA gene using V4-V5 domains from marine sponge Callyspongia sp. DNA sample (1: sample, M: Marker).

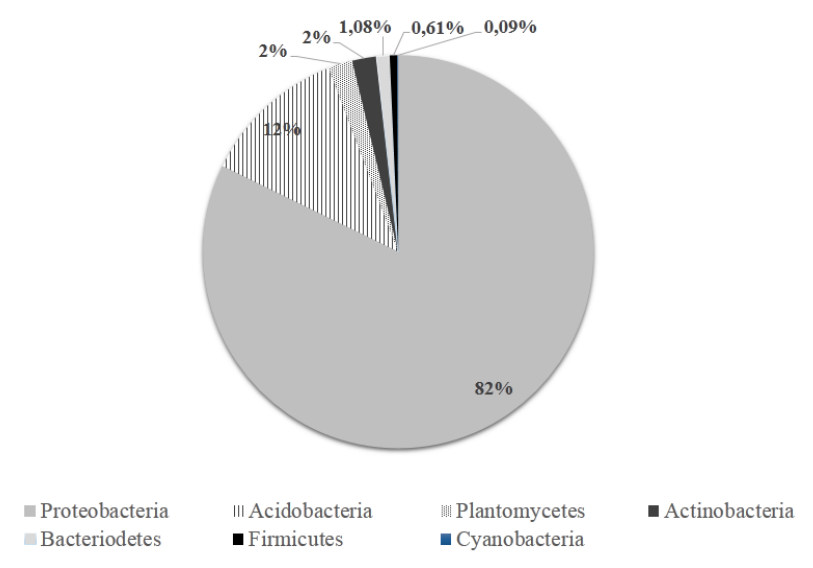

Figure 2. Diversity of bacteria community associated with marine sponge Callyspongia sp. at the phylum level 


\section{Sponge-associated actinobacteria community}

The results showed that actinobacteria community structure associated with Callyspongia sp. varied in the class levels, i.e Actinobacteria, Coriobacteria, and Thermoleophilia with the abundance of $83 \%, 16 \%$, and $1 \%$, respectively (Figure 3). Furthermore, on the basis of the order level, Actinobacteria was divided into three groups, i.e Actinomycetales (83\%), Coriobacteriales $(16 \%)$, and Gaielalles $(1 \%)$. Actinobacteria observed were also classified into 14 different families with the most dominant family was Microbacteriaceae (71\%), followed by Coriobacteriaceae (16\%), Micrococcaceae (7\%), Corynebacteriaceae (6\%), Propionibacteriaceae (5\%), Dermabacteriaceae (4\%), Geodermatophilaceae (1\%), Mycobacteriaceae (1\%), Nocardiaceae (1\%), Nocardioidaceae $(1 \%)$, and Gaiellaceae $(1 \%)$. The other phyla with a relative abundance below $1 \%$ were Streptomycetaceae $(0.90 \%)$, Williamsiaceae $(0.70 \%)$, and Pseudonocardiaceae $(0.45 \%)$ (Figure 4$)$.

\section{Discussion}

Successful DNA extraction for obtaining good DNA purity was achieved. Sponge samples had high moisture content with concentrated liquid colors, thus it can reduce the reagents sensitivity and effectiveness used during the extraction process. The recovery of high quality and high purity of the representative DNA from environmental samples is very important. Therefore, the extraction efficiency of microbial DNA from the initial samples is certainly important for determining its quality (Kennedy et al. 2014; Fang et al. 2015).

The results showed that there were 7 different phyla sponge-associated and Proteobacteria known as a dominant phylum. A previous study indicated that Proteobacteria was the dominant phylum associated with Callsypongia vaginalis (Bibi et al. 2019). Callyspongia sp. is included as Low Microbial Abundance sponges (LMA), recognized by the same group of Proteobacteria (Weisz et al. 2007; Giles et al. 2013). Proteobacteria perform different functions in host such as nitrogen fixation, host defense mechanism, and nitrification process (the oxidation of ammonia to nitrite) (Mohamed et al. 2010).

Acidobacteria has an important ecological role and extensive metabolic versatility in marine environment. The other phylum was found associated with Callyspongia was Planctomycetes. According to Sanchez et al. (2014), members of the genus Acidobacteria are very difficult to be cultured and currently available information is based on the 16S rRNA gene sequence. The genus Acidobacteria was also found to be associated with the sponge Palthoa australiae by $6 \%$ (Sun et al. 2014). They have important ecological functions in different marine environments, as shown to perform anaerobic ammonia oxidation (anammox). However, the anammox process is important in biological nitrogen removal from water containing high nitrogen and carbon ratios (Jeske et al. 2013).

Planctomycetes are a group of bacteria with the ability to produce various bioactive compounds. For instance Streptomyces, this genus has a large genome for a prokaryotic organism and a complex life cycle (Jeske et al. 2013). Planctomycetes are slow-growing bacteria, in their adaptation to various habitats including extreme environments this group of bacteria has developed an adaptation mechanism that is still unknown (Graca et al. 2016). Members of the Planctomycetes group have quite important ecological functions in the marine environment, namely through anaerobic ammonium oxidation (anammox). This anammox process is important to reduce the availability of biological nitrogen from water with high carbon and nitrogen content.

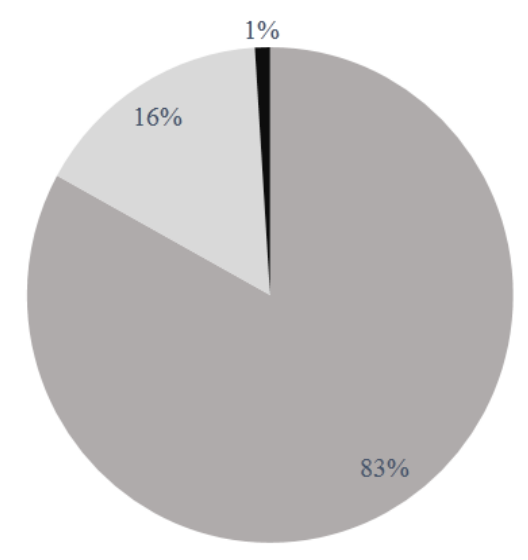

Actinobacteria Coriobacteria $\square$ Thermoleophillia

A

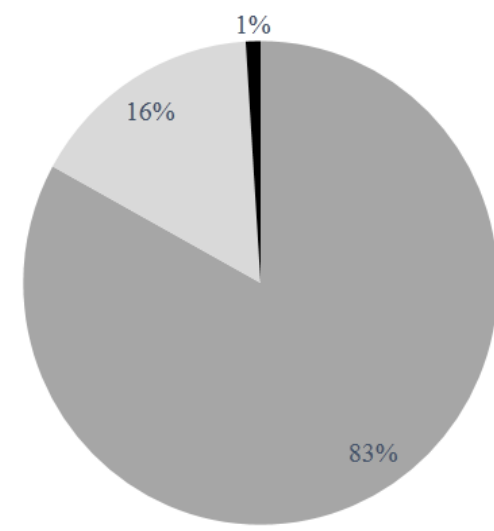

Actinomycetales Coriobacteriales a Gaiellales

\section{B}

Figure 3. The existence of actinobacteria community associated with marine sponge Callyspongia sp.; A. At the Class level, B. At the Order level. 


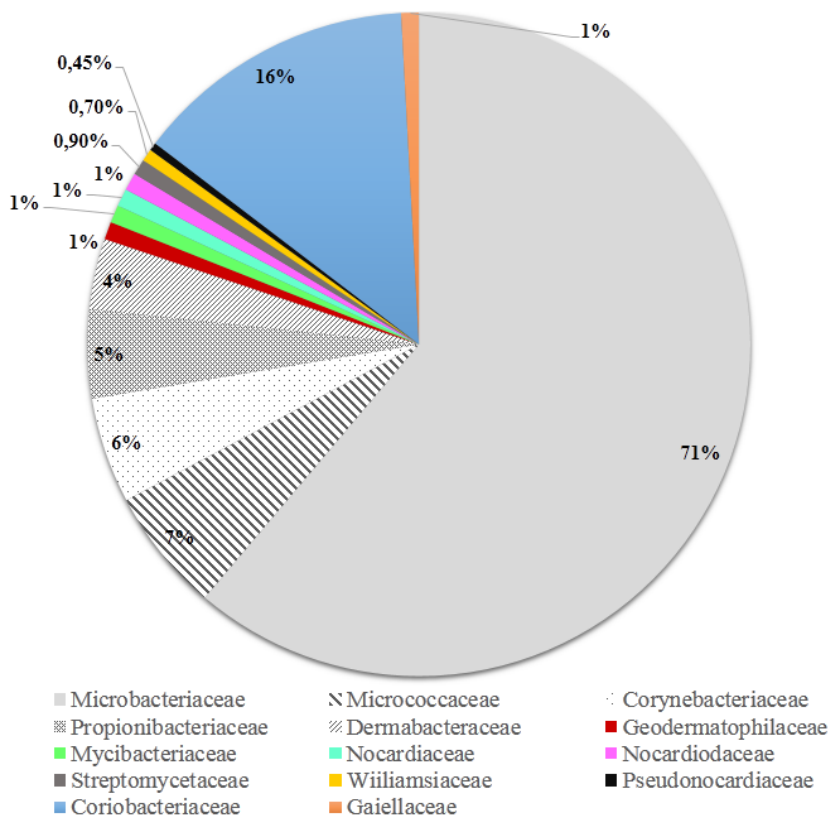

Figure 4. Diversity of the actinobacteria community associated with marine sponge Callyspongia sp. at the family level

The association of bacteria with the sponge plays an important role in the metabolism of carbon, nitrogen, and the production of secondary metabolites from the sponge as their host (Steinert et al. 2019; Fieth et al. 2015; Kennedy et al. 2014). Moreover, the role of associated bacteria plays important in the growth and development of sponges such as UV protection, defense against toxic compounds, stabilizing their skeletons, and decomposing residuals metabolites (Shick and Dunlap 2002). The different sponge contains bacterial communities consisting of mainly different bacterial species. This was suspected due to vertical transfer of species-specific bacterial communities.

Sponges are sessile and lack other anti-predator defense, secondary metabolites of bacteria can provide them with chemical defense the expression of secondary metabolites during predation may be independent of antibiotic expression in pure culture, the latter to have evolved for mutualism with higher animal of plant hosts (Harir et al 2018; Van der Meij et al. 2017). Sponges are a reservoir of exceptional microbial diversity and a major contributor to the total microbial diversity found in the world's ocean. Microbial communities exhibit little similarity in species composition or structure although a number of emerging properties related to community organization are evident (Thomas et al. 2016). The sponge host provides a special habitat for its symbiotic microbiota, and the microbial processes in the sponge holobiont have an impact on the health of the host and the surrounding ecosystem.

The investigation of Callyspongia sp. spongeassociated actinobacteria has successfully been conducted using NGS technology and identified 14 actinobacterial families, i.e. Microbacteriaceae, Micrococcaceae, Corynebacteriaceae, Propionibacteriaceae, Dermabacteriaceae, Geodermatophilaceae, Mycobacteriaceae, Nocardiaceae,
Coriobacteriaceae, Gaiellaceae, Nocardioidaceae, Streptomycetaceae, Williamsiacea, and Pseudonocardiaceae. The most dominant Callyspongia sp. sponge-associated actinobacteria family is Microbacteriaceae. The results of this study indicated that by using a metagenomic approach, the diversity of actinobacteria associated with the sponge Callyspongia sp. is more diverse when compared to cultivation techniques. The NGS technique has successfully described the diversity of actinobacteria that may not be detected using other molecular methods.

In this study, 14 families of microbes were obtained, being the dominant family was Microbacteriaceae. A previous report using Restriction Fragment Length Polymorphism (RFLP) method in Callyspongia sp. showed that this sponge has closed association with actinobacteria communities originated from the Micrococcaceae family (Qian et al. 2006). On the contrary, on the basis of DGGE method conducted by Yang et al. (2013), actinobacteria associated with Callyspongia sp. from Botany Bay (Sydney, Australia) were undetected. However, Callyspongia sp. from South Pacific Ocean resulted in, 4 bands that belonged to actinobacteria phylum. Sequencing results of the four bands were uncultured actinobacteria EU816864, uncultured actinobacterium FN556247, uncultured actinobacterium FN556246, and uncultured actinobacterium FN556248 (Park 2010). The DGGE method only describes the dominant species found in the sample, whereas RFLP result may be interfered with a PCR bias and gives complicated bands. Meanwhile, using NGS technology will read all the genomes of the DNA template randomly, thereby that the entire bacterial community contained in the sample will be completely detected. The use of different methods will affect to the community of sponge-associated actinobacteria. 
Xestospongia sp. which is the same order as Callyspongia i.e. Haplosclerida was reported to have an association with Micromonospora, Streptomyces, Saccharomonospora, and Verrucosispora (Vicente et al. 2013). Actinobacteria found in Halicondra sp. which is the same family as Callyspongia sp. was Demospongia. There are five genera of actinobacteria associated with that sponge i.e. Actinoalloteichus, Micrococcus, Micromonospora, Nocardiopsis, and Streptomyces. Furthermore, Simamora et al. (2016) found five genera of sponge-associated actinobacteria with Neofibularia sp. detected by DGGE method which was identified as rare actinobacteria, i.e. Ferrithrix, Lamia, Aciditerrimonas, Thermobispora, and Propionibacterium.

The difference in actinobacteria diversity in sponge samples is caused by numerous factors, such as nutrients and environmental factors. Sponge-associated actinobacteria required simple nutrients available in the environment. Notably, the sponge contains a diverse actinobacteria group, however, their ecological functions are hardly known. Sponge-associated actinobacteria were reported could produce bioactive substances that may play an important role in the chemical ecology of sponge hosts (Sun et al. 2015). In addition to environmental factors, nutrition also affects the diversity of actinobacteria associated with certain sponges. Actinobacteria have the ability to break down complex compounds into simpler compounds to be used as nutrients for their growth. The existence of anthropogenic interventions will cause changes in the actinobacterial community associated with certain sponges (Undabarrena et al. 2016).

Actinobacteria might play a major role in the defense system of the life cycle in marine sponges (Bibi et al. 2019). A total of $74 \%$ of sponge-associated actinobacteria showed antagonism against assayed pathogenic bacteria (Liu et al. 2019). In addition, members of actinobacteria phylum might also be able to synthesize polyketides (bioactive compounds) in a marine sponge, indicating that they also played an important role in sponge defense systems against predators. The sponge is limited to innate immunity. They only employ physiochemical barriers, such as mucous layers, which act as sponge cellular defense. Interestingly, a lot of sponge-associated actinobacteria are known to have the potential symbiotic relations and/or could produce secondary metabolite productions. Several genera of actinobacteria i.e Acidimicrobium, Brachbacterium, Corynebacterium, Leucobacter were found in various sponge species (Khan et al. 2012).

In 2013, the diversity of actinobacteria associated with sponges was constructed using a phylogenetic tree with a sequence length of $1300 \mathrm{bp}$. There were 60 different actinomycete genera known to be associated with sponges. The suborder Micrococcineae is known to be the most suborder found in sponges. The most common genera are Microbacterium, Micrococcus, and Arthrobacter. Marine sponges are also known to have the ability to associate with rare actinomycetes such as Actinokineospora, Actinomadura, Amycolaptosis, Knoellia, Nonomuraea, Pseudonocardia, Saccharomonospora, Saccharopolyspora, Verrucosispora which are thought to have the ability to produce novel metabolites. Several new species of actinomycete strains associated with sponges have been found, such as Streptomyces axinellae sp. nov. associated with the sponge Axinella polypoides, Saccharopolyspora cebuensis sp. nov. isolated from the sponge Haliclona sp. from the waters of Cebu, Philippines, and Actinoalloiteichus hymeniacidonis sp. nov. from the sponge Hymeniacidon perleve from Chinese waters. Three new actinomycetes species namely Streptomyces tateyamensis sp. nov., S. marinus sp. nov., and S. haliclonae sp. nov., were successfully isolated from the sponge Haliclona sp. located in Tateyama City, Japan (Hentschel et al. 2012).

Culturable sponge-associated actinobacteria from Callyspongia sp. have been reported by (Retnowati et al. 2018). A total of 18 actinobacterial isolates were obtained by using a HVA medium. The largest number of actinobacteria members which was successfully isolated was Streptomyces. Phylogenetic analysis based on $16 \mathrm{~S}$ rRNA gene sequence indicated that five culturable actinobacterial isolates were highly similar to Streptomyces sp., Streptomyces rubrogriseus AJ781373, Streptomyces tendae D63873, Streptomyces violaceorubidus AJ781374, and Streptomyces tritolerans DQ345779 (Retnowati et al. 2019). Intriguingly, in comparison of the findings with those of our recent studies confirms, the diversity of Streptomyces was only $0.9 \%$ of the total diversity (Figure 3 ). This study supports the evidence from previous studies (Abdelmohsen et al. 201o, 2014), that Streptomyces is a common genus of sponge-associated actinobacteria. This means that Streptomyces is the most actinobacterial genusoften found associated with various types of sponges observing by both culturable and metagenomic techniques.

Streptomyces spp. are not only benefiting from the resources of their hosts, and this interaction controls the expression of secondary metabolites produced by the actinobacteria. The exposure of the endosymbiont Streptomyces ACT-52A to Applysilla resoa promoted production of bioactive compounds with antibacterial activity (Mehbub et al. 2016). The factors involved in controlling the expression of these smBGCs are likely to be quite diverse, given the large degree of variability in the habitats and potential hosts, and how they are presumably influencing the secondary metabolite biosynthetic potential of Streptomyces symbionts (Adnani et al. 2017). Moreover, secondary metabolites from marine actinomycetes usually show distinct and often complex chemical structures, which help the development of further therapeutic agents efficiently (Elsayed et al. 2021). Molecular-based culture methods by adding nutrient supplements that mimic the marine conditions in nutrients content, oxygen gradient, $\mathrm{pH}$, etc. have been highly efficient in overcoming technical bias and maximize the cultivable efficiency in recent times (Trindade et al. 2015).

The research showed that NGS technology can successfully reveal rare actinobacteria groups associated with the Callyspongia sp. that might have been undetected by culturable approach. Among all taxa, bacteria from the order actinomycetes are the active substance producers of marine microbial communities that form a stable and persistent population across different marine ecosystems 
(Mahapatra et al. 2019). For years, actinobacteria were isolated from aquatic sources with limited usages. However, the introduction of metagenomic techniques in recent times has considerably boosted the genomic sequencing of marine actinobacteria exploring their huge biosynthetic potential from marine and other sources (Prichystal et al. 2016).

The use of NGS technology for describing microbial diversity has greatly enhanced the knowledge of community structure in sponge-associated actinobacteria. Supporting these results, the use of V4 and V5 domains showing the conservation and the hypervariable region of this NGS method could provide comprehensive importance on microbial community in sponges. The Miseq Illumina platform will produce more reads with a shorter sequence length of 250-300 bp. The length of the resulting sequence is not as long as the sequence produced by conventional sequencing, but the length of the sequence is quite feasible to be used in the taxonomic analysis (Vasileiadis et al. 2012). In addition, the quality of the resulting sequence is also higher than other platforms, so that the nucleotide that is read is more reliable.

\section{ACKNOWLEDGEMENTS}

We gratefully thank the Ministry of Research, Technology and Higher Education of Indonesia for the funding support.

\section{REFERENCES}

Abdelmohsen U, Pimentel-Elardo SM, Hanora A, Radwan M, AbouEl-Ela SH, Ahmed S, Hentschel U. 2010. Isolation, phylogenetic analysis and antiinfective activity screening of marine sponge-associated actinomycetes. Mar Drugs 8 (3): 399-412. DOI: 10.3390/md8030399.

Abdelmohsen U, Yang C, Horn H, Hajjar D, Ravasi T, Hantscel U. 2014. Diversity, abundance and natural products of marine spongeassociated actinomycetes. Nat Prod Rep 31 (3): 381-399. DOI: 10.1039/C3NP70111E

Bibi F, Alvi SA, Al-Sofyani A, Naseer MI, Yasir M, Azhar EI. 2019 Pyrosequencing reveals sponge-specific bacterial communities in marine sponges of Red Sea, Saudia Arabia. Saudi J Biol Sci 27 (1): 67-73. DOI: 10.1016/j.sjbs.2019.05.002.

Bokulich NA, Joseph ML, Allen G, Benson AK, Mills DA. 2012. NextGeneration Sequencing reveals significant bacterial diversity of botrytized wine. PLoS ONE 7 (5): e36357. DOI: 10.1371/journal.pone.0036357.

Elsayed Y, Fahim JR, Abdelmohsen UR, Fouad MA. 2021. Chemical and biological investigation of the marine bacterium Rhodococcus sp UA13. J Adv Biomed Pharm Sci 4 (1): 1-6. DOI: 10.21608/jabps.2020.38170.1094.

Fang Y, Xu M, Chen X, Sun G, Guo J, Wu W, Liu X. 2015. Modified pretreatment method for total microbial DNA extraction from contaminated river sediment. Front Chem Sci Eng 9 (3): 444-452. DOI: $10.1007 / \mathrm{s} 11783-014-0679-4$.

Fieth RA, Gauthier MA, Bayes J. 2015. Ontogenetic changes in the bacterial symbiont community of the tropical demosponge Amphimedon queenslandica: Metamorphosis is a new beginning. Front Mar Sci 3: 228. DOI: 10.3389/fmars.2016.00228.

Gelani CD, Uy. 2016. Cytotoxicity to Artemia salina L. of marine sponge extracts from Surigao del Norte, Philippines. Bull Env Pharmacol Life Sci 5 (5): 14-18.

Giles EC, Kamke J, Moitinho S, Taylor MW, Hentschel U, Ravasi T, Schmitt S. 2013. Bacterial community profiles in low microbial abundance sponges. FEMS Microbiol Ecol 83 (1): 232-241. DOI: 10.1111/j.1574-6941.2012.01467.x

Graca AP, Calisto R, Lage OM. 2016. Planctomycetes as novel source of bioactive molecules. Front Microbiol 7: 1241. DOI: 10.3389/fmicb.2016.01241.

Harir M, Bendif H, Bellahcene M, Fortas Z, Pogni R. 2018. Streptomyces 523 secondary metabolites in Basic Biology and Applications of Actinobacteria. IntechOpen, London. DOI: 10.1101/2020.01.09.901108.

Hentschel U, Schmid M, Wagner M, Fieseler L, Gernert C, Hacker J. 2012. Isolation and phylogenetic analysis of bacteria with antimicrobial activities from the Mediterranean sponges Aplysina aerophoba and Aplysina cavernicola. FEMS Microbiol Ecol 35 (3): 305-312. DOI: 10.1111/j.1574-6941.2001.tb00816.x.

Jeske O, Jogler M, Petersen J, Sikonski J, Jogler C. 2013. From genome mining to phenotypic microarrays: Planctomycetes as source for novel bioactive molecules. Anton Leeuw Intl J 104: 551-567. DOI: 10.1007/s10482-013-0007-1.

Kapojos MM, Abdjul DB, Yamazaki H, Ohshiro T, Rotinsulu H, Wewengkang DS, Sumilat DA, Tomoda H, Namikoshi M, Uchida R. 2018. Callyspongiamides A and B, sterol O-acyltransferase inhibitors, from the Indonesian marine sponge Callyspongia sp. Bioorg Med Chem Lett 28 (10): 1911-1914. DOI: 10.1016/j.bmcl.2018.03.077.

Kennedy J, Flanner B, Jackson SA, Morrissey JP, O'Gara F, Dobson ADW. 2014. Evidence of a putative deep sea-specific microbiome in marine sponges. PLoS ONE 9 (7): e103400. DOI: 10.1371/journal.pone.0091092

Khan ST, Takagi M, Shin-ya K. 2012. Actinobacteria associated with marine sponge Haliclona sp.; Biosynthetic genes for secondary metabolites and product. Microbes Environ 13 (2): 391-403. DOI: 10.1007/s13213-013-0652-7.

Liu T, Wu S, Zhang R, Wang D, Chen J, Zhao J. 2019. Diversity and microbial potential of Actinobacteria isolated from diverse marine sponges along the Beibu Gluf of the South Sea. FEMS J 95 (7): 1-10. DOI: $10.1093 /$ femsec/fiz089.

Mahapatra GP, Raman S, Nayak S, Gouda S, Das G, Patra JK. 2019. Metagenomics approaches in discovery and development of new bioactive compounds from marine Actinomycetes. Curr Microbiol 77 (4): 645-656. DOI: 10.1007/s00284-019-01698-5.

Menbub MF, Tanner JE, Barnett SJ, Franco CMM, Zhang W. 2016. The role of sponge-bacteria interactions: The sponge Aplysilla rosea challenged by its associated bacterium Streptomyces ACT-52A in a controlled aquarium system. Appl. Microbiol Biotechnol 100: 1060910626. DOI: $10.1007 / \mathrm{s} 00253-016-7878-9$

Mohamed NM, Saito K, Tal Y, Hill RT. 2010. Diversity of aerobic and anaerobic ammonia-oxidizing bacteria in marine sponges. ISME J 4 (1): 38-48. DOI: 10.1038/ismej.2009.84.

Park JS. 2010. Bacterial community diversity associated with two marine sponges from the South Pacific Ocean based on 16S rDNA-DGGE analysis. Korean J Microbiol 46 (3): 255-261. DOI: 10.1007/s00248006-9177-y.

Prichystal J, Schug KA, Lemr K, Novak J, Havlicek V. 2016. Structure analysis of natural products. Anal Chem 88 (21): 10338-10346. DOI: 10.1021/acs.analchem.6b02386.

Qian PY, Dobretsov S, Dahms UH, Pawlik J. 2006. Antifouling activity and microbial diversity two congeneric sponges Callyspongia spp. from Hongkong and the Bahamas. Mar Ecol Prog Ser 324: 151-165. DOI: $10.3354 /$ meps 32415 .

Trindade M, Vanzyl LJ, Navarro-Fernández J, Elrazak A 2015. Targeted metagenomics as a tool to tap into marine natural product diversity for the discovery and production of drug candidates. Front Microbiol 6: 890. DOI: 10.3389/fmicb.2015.00890.

Retnowati D, Solihin DD, Ghulamahdi M, Lestari Y. 2019. Characterization of sponge-associated actinobacteria with potential to promote plant growth in tidal swamps. J Biol Res 92 (8191): 65-71. DOI: $10.4081 /$ jbr.2019.8191.

Retnowati D, Solihin DD, Ghulamahdi M, Lestari Y. 2018. New information on the potency of sponge-associated actinobacteria as producer of plant growth-promoting bioactive compounds. Malays Appl Biol 47 (6): 127-135..

Shick JM, Dunlap WC. 2002. Mycosporine-like amino acids and related gadusols: Biosynthesis, acumulation, and UV-protective functions in aquatic organism. Ann Rev Physiol 64: 223-262. DOI: 10.1146/annurev.physiol.64.081501.155802.

Silva LM, Nielsen S, Amir A, Gonzalez A, Ackermann GL, Cerrano C, Garcia CA, Easson C, Sipkema D, Liu F. 2017. The sponge 
microbiome project. GigaScience 6 (10): 1-7. DOI 10.1093/gigascience/gix077.

Simamora CJK, Slihin DD, Lestari Y. 2016. Culturable and unculturable actinomycetes associated with the sponge Neofibularia from Bira Island, Indonesia. Malays J Microbiol 12 (3): 211-220. DOI: $10.21161 / \mathrm{mjm} .77015$

Steinert G, Wemheuer B, Janussen D, Erpenbeck D, Daniel R, Simon M, Brinkhoff T, Schupp PJ. 2019. Prokaryotic diversity and community patterns in a antartic continental shelf sponges. Front Mar Sci 6 (297): 1-15. DOI: $10.3389 /$ fmars.2019.00297.

Sun W, Zhang F, He L, Karthik L, Li Z. 2015. Actinomycetes from the South China Sea sponges: Isolation, diversity, and potential for aromatic polyketides discovery. Front Microbiol 8 (6): 1-15. DOI: 10.3389/fmicb.2015.01048.

Sun W, Zhang F, He L, Li Z. 2014. Pyrosequencing reveals diverse microbial community associated with the Zoanthid Palythoa australiae from the South China sea. Microb Ecol 67 (4): 942-950. DOI: 10.1007/s00248-014-0395-4.

Thomas T, Moitinho-Silva L, Lurgi M, Björk JR, Easson C, AstudilloGarcía C. 2016. Diversity, structure and convergent evolution of the global sponge microbiome. Nat Commun 7: 11870. DOI: 10.1038/ncomms 11870 .
Undabarrena A, Beltrametti F, Claverias FP, Gonzaez M, Moore ERB, Seeger M, Camara B. 2016. Exploring the diversity and antimicrobial potential of marine actinobacteria from the Comau Fjord in Nirthern Patagonia, Chile. Front Microbiol 7: 1135. DOI: 10.3389/fmicb.2016.01135.

Van der Meij A., Worsley SF, Hutchings MI, Van Wezel GP. 2017. Chemical ecology of antibiotic production by actinomycetes. FEMS Microbiol Rev 41: 392-416. DOI: 10.1093/femsre/fux005.

Vicente J, Stewart A, Song B, Hill RT, Wright JL. 2013. Biodiversity of Actinomycetes associated with caribbean sponges and their potential for natural product discovery. Mar Biotechnol 15 (4): 413-424. DOI: 10.1007/s10126-013-9493-4.

Weisz JB, Hentschel U, Lindquist N, Martens CS. 2007. Linking abundance and diversity of sponge-associated microbial communities to metabolic differences in host sponges. Mar Biol 152 (2): 475-483. DOI: $10.1007 / \mathrm{s} 00227-007-0708-y$.

Yang CL, Wang YS, Liu CL, Zeng YJ, Cheng P, Jiao RH. 2017. Strepchazolins A and B: Two new alkaloids from a marine Streptomyces chartreusis NA02069. Mar Drugs 15 (8): 244. DOI: 10.3390/md15080244. 